\title{
EZH2 silencing with RNAi enhances irradiation-induced inhibition of human lung cancer growth in vitro and in vivo
}

\author{
HUI XIA, CHANG-HAI YU, YIMING ZHANG, JIANQI YU, JIE LI, WEN ZHANG, \\ BAOSHI ZHANG, YINGJIE LI and NANNAN GUO
}

\author{
Department of Cardiothoracic Surgery, First Affiliated Hospital of PLA General Hospital, Beijing 100048, P.R. China
}

Received February 5, 2012; Accepted April 18, 2012

DOI: $10.3892 / \mathrm{ol} .2012 .696$

\begin{abstract}
Non-small cell lung cancer (NSCLC) has a high mortality rate and poor prognosis. The aim of the present study was to silence EZH2 and explore the antitumor effect of small interfering RNA (siRNA)-EZH2 in combination with radiotherapy, which is a main treatment for NSCLC. The results showed that irradiation in the presence of siRNAEZH 2 arrested A549 cells in the $\mathrm{G}_{0}$ and $\mathrm{G}_{1}$ phases, delayed cell cycle progression and effectively inhibited cell proliferation, compared with cells that received radiotherapy alone. The combined therapy enhanced the percentage of apoptotic A549 cells in vitro and reduced the tumor size, in addition to increasing the survival rate in tumor xenograft experiments. This study demonstrates the antitumor activity of ionizing radiation therapy in combination with siRNA-EZH2 in NSCLC, both in vitro and in vivo, as well as providing a scientific rationale for targeting EZH2 to enhance the sensitivity of cancer to radiotherapy in NSCLC patients.
\end{abstract}

\section{Introduction}

As a result of poor prognosis, lung cancer is a major cause of cancer-related mortality. Non-small cell lung cancer (NSCLC) accounts for approximately $80 \%$ of all lung cancer cases and has a 5-year overall survival rate of less than $15 \%(1,2)$. Approximately $40 \%$ of patients diagnosed with NSCLC have unresectable stage III disease or medically inoperable disease (3). Although there have been recent advances and developments in numerous therapeutic strategies, including surgery, chemotherapy and radiotherapy, poor survival remains an issue due to the high systemic toxicity and drug resistance. Therefore, the development of novel approaches for the diagnosis, treatment and prevention of NSCLC, including targeted

Correspondence to: Dr Chang-Hai Yu, Department of Cardiothoracic Surgery, First Affiliated Hospital of Chinese PLA General Hospital, Beijing 100048, P.R. China

E-mail: yuchanghai1@hotmail.com

Key words: lung cancer, ionizing radiation, EZHE2, small interfering RNA, cooperative gene treatment as an adjuvant modality or as a radiosensitizer to treat this lethal disease, is urgently needed to enhance the survival rate in patients.

One of the targets currently being evaluated for the treatment of lung cancer is EZH2. EZH2 is a catalytically active component of the PRC2 complex, which is a human homolog of the Drosophila protein 'Enhancer of Zest'. EZH2 is involved in the transcriptional repression of specific genes by the trimethylation of lysine 27 and, to a lesser extent, lysine 9 of histone H3. EZH2 contains a SET domain with an intrinsic histone lysine methyltransferase activity and directly interacts with and regulates the activity of the DNA methyltransferases (DNMTs) DNMT1, DNMT3a and DNMT3b. Various studies have found that the abnormal expression of $\mathrm{EZH} 2$, a potential marker used to distinguish aggressive from indolent or benign cancers, is involved in the tumorigenesis of several malignancies, including melanoma, prostate, breast, bladder and endometrial cancers (4). EZH2 also provides proliferative advantages to eukaryotic cells through interaction with pathways of key elements that control cell growth arrest and differentiation (4-6). As a transcriptional repressor, EZH2 is involved in controlling cell growth and proliferation by promoting the S-phase entry and $\mathrm{G}_{2} / \mathrm{M}$ transition. EZH2 also promotes the repression of certain genes. This involves histone deacetylation by histone deacetylase-1 (HDAC-1) with which EZH2 interacts through its PRC2-binding partner EED $(7,8)$.

Recently, the successful use of small interfering RNA (siRNA) to downregulate gene expression in several model systems has led to an increasing number of attempts to explore this methodology in a potentially therapeutic setting. The knockdown of EZH2 by siRNA inhibits breast cancer cell proliferation, whereas the pharmacological inhibition of EZH2 results in the apoptosis of breast cancer cells (9). At present, few studies concerning NSCLC and its response to radiotherapy treatment in combination with silencing EZH2 using siRNAs have been published. Therefore, combining siRNA-EZH2 targeting with irradiation has potential as a therapeutic option.

In the present study, we aimed to silence EZH2 and explore the antitumor effect of siRNAs on lung cancer when applied in combination with radiotherapy. We hypothesized that the inhibition of the EZH2-mediated signal transduction pathway using siRNA is an effective strategy to increase the sensitivity of NSCLC patients to radiotherapy. 


\section{Materials and methods}

Cell lines and culture. The human lung adenocarcinoma A549 and HTB-56 cell lines were obtained from the American Type Culture Collection (ATCC, Manassas, VA, USA). The cells were maintained in Dulbecco's modified Eagle's medium (DMEM, Gibco, Carlsbad, CA, USA) and supplemented with $10 \%$ heat-inactivated FBS and $1 \%$ penicillin/streptomycin at $37^{\circ} \mathrm{C}$ in a humidified incubator with $95 \%$ air and $5 \% \mathrm{CO}_{2}$.

siRNA transfection. A549 and HTB-56 cells were plated on six-well plates at a density of $2 \times 10^{5}$ cells/well and grown overnight until $50-80 \%$ confluence was achieved to obtain maximum transfection efficiency. The cells were transfected with validated siRNA for EZH2 and a negative control vector (Qiagen, Lafayette, CO, USA) at a concentration of $100 \mathrm{nM}$ using Lipofectamine 2000 transfection reagent (Invitrogen, Carlsbad, CA, USA) according to the manufacturer's instructions. The medium was replaced with standard culture medium $6 \mathrm{~h}$ post-transfection.

Irradiation treatment. On the days after siRNA transfection, sub-confluent cell monolayers were treated with $\gamma$-ray irradiation from a ${ }^{60} \mathrm{Co}$ source (PLA General Hospital, Beijing, China) at $\sim 2 \mathrm{~Gy} / \mathrm{min}$ every 3 days for 2 weeks.

Western blot analysis. To assess EZH2 expression, $30 \mu \mathrm{g}$ of total cell protein extracts were obtained from A549 and HTB-56 cells following centrifugation at $12,000 \mathrm{x}$ g for $30 \mathrm{~min}$ at $4^{\circ} \mathrm{C}$. The cells were lysed in buffer containing $20 \mathrm{mM}$ Tris- $\mathrm{HCl}$ ( $\mathrm{pH} 7.5$ ), $150 \mathrm{mM} \mathrm{NaCl}, 1 \mathrm{mM}$ EDTA, $1 \mathrm{mM}$ EGTA, $1 \%$ Triton X-100, $2.5 \mathrm{mM}$ sodium pyrophosphate, $1 \mathrm{mM} \beta$-glycerophosphate, $1 \mathrm{mM} \mathrm{Na} \mathrm{VO}_{4}, 1 \mu \mathrm{g} / \mathrm{ml}$ leupeptin supplemented with proteinase (Roche Diagnostics, Mannheim, Germany) and phosphatase inhibitor cocktails (Sigma, St. Louis, MO, USA). The protein concentrations of cell lysates were determined using a BCA protein assay kit (Pierce Biotechnology, Inc., Rockford, IL, USA). The cell lysates were separated by SDS-PAGE and analyzed according to standard western blotting procedures. After transferring to PVDF membranes and probing with 1:1,000-diluted anti-EZH2 primary antibodies (Cell Signaling Technology, Beverly, MA, USA) and $\beta$-actin, the membranes were incubated with horseradish peroxidase-conjugated secondary antibody and visualized with ECL reagents (Pierce Biotechnology, Inc.).

Cell proliferation. Cell proliferation was assayed using a cell proliferation MTT [3-(4,5-dimethylthiazol-2-yl)2,5-di-phenyltetrazolium bromide] kit (Roche Diagnostics) according to the manufacturer's instructions. A549 and HTB-56 cells were seeded in triplicate onto 96-well tissue culture plates at a density of $5 \times 10^{3}$ cells/well. The cells were transfected with siRNA-EGFR and treated with irradiation at $\sim 2 \mathrm{~Gy} / \mathrm{min}$. Cell proliferation was determined by assessing the mitochondrial reduction of MTT. Cell growth was analyzed on a plate reader at a wavelength of $570 \mathrm{~nm}$ using a Universal Microplate Spectrophotometer (BioTek Instruments, Winooski, VT, USA). Experiments were performed in triplicate.
Cell cycle analysis by flow cytometry. For the cell cycle analysis, A549 and HTB-56 cells were plated at $1 \times 10^{5}$ cells/ well on six-well plates. Following treatment with siRNA and irradiation, cells from each group were harvested with $0.125 \%$ trypsin, washed twice with ice-cold PBS and fixed with $70 \%$ (v/v) ethanol overnight at $4^{\circ} \mathrm{C}$. The cells were resuspended to a concentration of $1 \times 10^{6}$ cells $/ \mathrm{ml}$ in PBS and incubated with $100 \mu \mathrm{g} / \mathrm{ml} \mathrm{RNase}$ A and $50 \mathrm{mg} / \mathrm{ml}$ propidium iodide (PI) at room temperature for $30 \mathrm{~min}$. The distribution of the cells throughout the cell cycle was determined by flow cytometry (Becton Dickinson, San Jose, CA, USA) and analyzed with CellQuest software version 3.3 (Becton Dickinson). Each test was performed a minimum of three times.

Cell apoptosis analysis by flow cytometry. A549 and HTB-56 cells were seeded at a density of $1 \times 10^{5}$ cells/well and treated with irradiation in the presence or absence of siRNA-EZH2. To determine the apoptotic rate, the cells were recollected with PBS and stained with PI and Annexin V-FITC. Following staining, cell apoptosis was analyzed by flow cytometry.

In vivo tumor xenograft studies. A549 cells $\left(1 \times 10^{5}\right.$ cells/ animal) were subcutaneously injected into the left dorsal flank of 6- to 8-week-old female nude mice. After 2 weeks, the tumors reached $4-5 \mathrm{~mm}$ in diameter and the mice were randomly separated into three treatment groups ( $\mathrm{n}=8$ animals per group). Radiotherapy (2 Gy/dose every 3 days for 2 weeks) was administered to the animals in the presence or absence of siRNA-EZH2 (60 $\mu \mathrm{g} / \mathrm{dose})$. Control animals were injected with only PBS and all intratumoral injections were performed under anesthesia. Subcutaneous tumor growth was measured every third day and the mean tumor volume was calculated as width $^{2} \mathrm{x}$ length $\mathrm{x} 0.52$. The research protocol was approved by the Animal Ethics Committee of PLA General Hospital. Measurements were performed in a coded and blinded fashion. The therapeutic effect was determined by statistical analysis using the Student's t-test.

\section{Results}

Effective inhibition of cell proliferation following combination treatment. Using western blotting, we found that the transfection of siRNA-EZH2 into A549 and HTB-56 cells downregulated EZH2 expression by $\sim 87$ and $\sim 75 \%$, respectively (Fig. 1). In addition, the expression of EZH2 was markedly higher in A549 than in HTB-56 cells. In the subsequent experiments, we tested the hypothesis that cooperative anti-proliferative effects occur by combining siRNA-EZH2 treatment with ionizing radiation in A549 and HTB-56 cells. The two cell lines were irradiated with $2 \mathrm{~Gy} \gamma$-rays at different time points in the presence or absence of siRNA-EZH2. Following treatment, daily analysis of cell viability on days 1-5 demonstrated that the combined treatment significantly inhibited cell proliferation $(\mathrm{P}<0.001)$ in A549 and HTB-56 cells. The number of A549 and HTB-56 cells was reduced by 79.7 and $62.8 \%$, respectively, following irradiation in the presence of siRNA-EZH2. In cells that received only radiotherapy, the number of A549 and HTB-56 cells was reduced by 59.3 and $55.1 \%$, respectively (Fig. 2). 

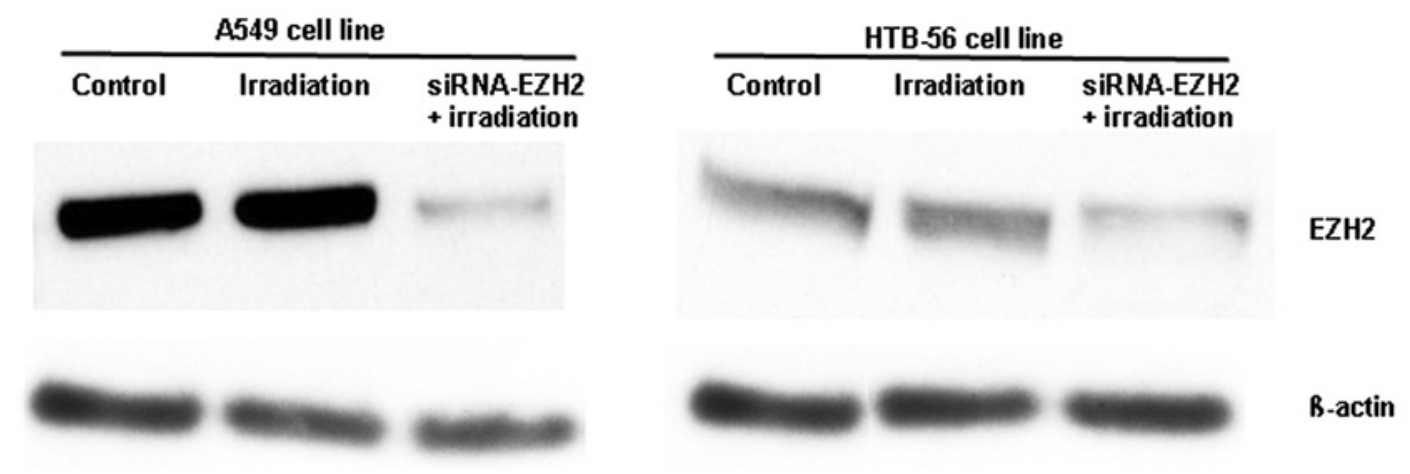

Figure 1. EZH2 protein expression was analyzed by western blotting. Lanes show the control group, cells treated with $\gamma$-ray irradiation at $\sim 2 \mathrm{~Gy} / \mathrm{min}$ and cells treated with irradiation in the presence of siRNA-EZH2. The corresponding $\beta$-actin levels are shown as a loading control. siRNA, small interfering RNA.
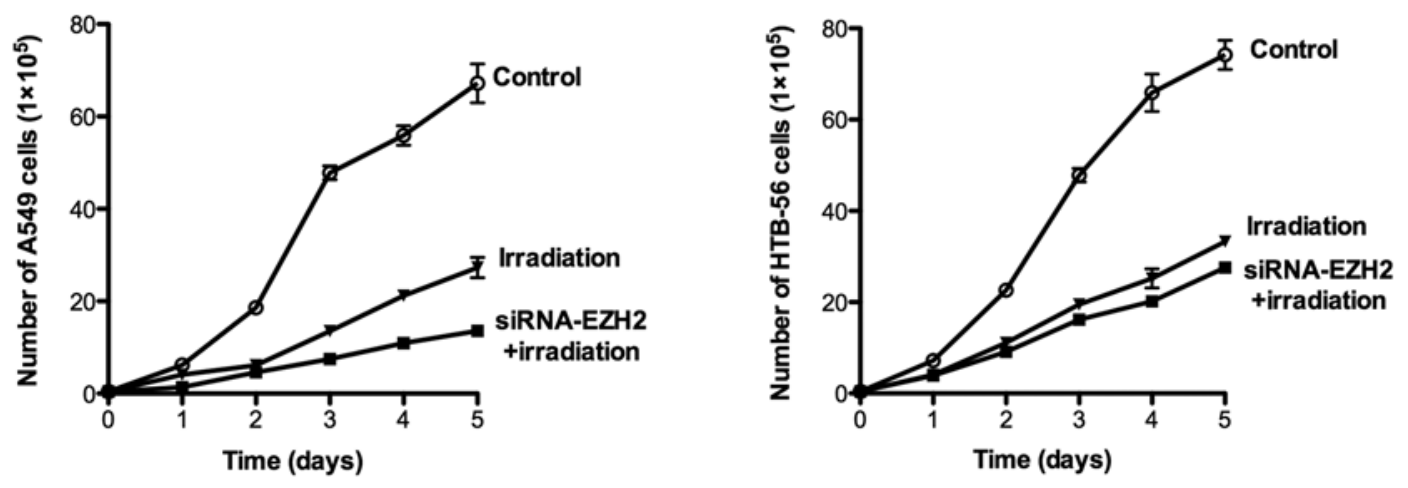

Figure 2. Effect of combination therapy on the growth of the A549 and HTB-56 human lung cancer cell lines in vitro. The cells were treated with $\gamma$-ray irradiation at $\sim 2 \mathrm{~Gy} / \mathrm{min}$ in the presence and absence of siRNA-EZH2. Data show the standard deviations of the mean. Each experiment was performed in triplicate. siRNA, small interfering RNA.

Effects of irradiation on the cell cycle in the presence of siRNA-EZH2. The cell cycle distribution of A549 and HTB-56 cells was investigated via FACS in the radiotherapy only and combination-treated groups. Following irradiation, the S-phase fraction of A549 and HTB-56 cells was altered in the presence and absence of siRNA-EZH2 (Fig. 3). The downregulation of EZH2 in A549 and HTB-56 cells for $48 \mathrm{~h}$ produced a 17 and $11 \%$ increase, respectively, in the number of cells in the $\mathrm{G}_{2} / \mathrm{M}$ phase following irradiation compared with the cells that received only radiotherapy. This result indicates that siRNAEZH2 arrested the cells in the $\mathrm{G}_{2} / \mathrm{M}$ phase, delaying cell cycle progression. Furthermore, the change caused by the siRNA differed between the A549 and HTB-56 cells, although they received the same treatment.

Effects on cell apoptosis following combined treatment. The results of the flow cytometry showed that irradiation treatment combined with siRNA-EZH2 induced changes in the percentage of apoptotic cells in A549 and HTB-56 cells compared with cells that received irradiation alone (Fig. 4). Some divergence in apoptosis was observed between the A549 and HTB-56 cells. The percentage of cells undergoing apoptosis in the A549 and HTB-56 cells was $14.35 \pm 1.05$ and $12.23 \pm 2.18 \%$, respectively, following irradiation with 2 Gy $\gamma$-rays, and $23.41 \pm 2.35$ and $15.35 \pm 3.14 \%$, respectively, following treatment with irradiation coupled with siRNA-EZH2. A significant difference was found between the irradiation-only and combination therapy groups $(\mathrm{P}<0.01)$.

Effects of combination therapy on subcutaneous tumor growth. When tumors were established 2 weeks after A549 cell injection, the nude mice were divided into three groups: a control group without any treatment and two groups treated with irradiation for 2 weeks in the presence and absence of siRNA-EZH2. The tumor growth and survival rates were evaluated following the application of the different therapeutic methods. The tumor size was measured 6 weeks later and was $2.1 \pm 0.03 \mathrm{~cm}^{3}$ in control animals, $0.7 \pm 0.04 \mathrm{~cm}^{3}$ following radiotherapy alone and $0.23 \pm 0.01 \mathrm{~cm}^{3}$ following combination therapy. Treatment with irradiation for 2 weeks induced the inhibition of tumor growth in all the mice (Fig. 5). Although complete tumor regression was not observed in the combination therapy and irradiation-only groups, treatment with siRNA-EZH2 and radiotherapy caused an increased inhibition of A549 tumor growth and increased the survival rate of tumor-bearing mice, thus, demonstrating the enhanced effect of the combined treatment in vivo.

\section{Discussion}

For most NSCLC patients, radiation therapy is crucial in the treatment of the disease. However, long-term survival of 

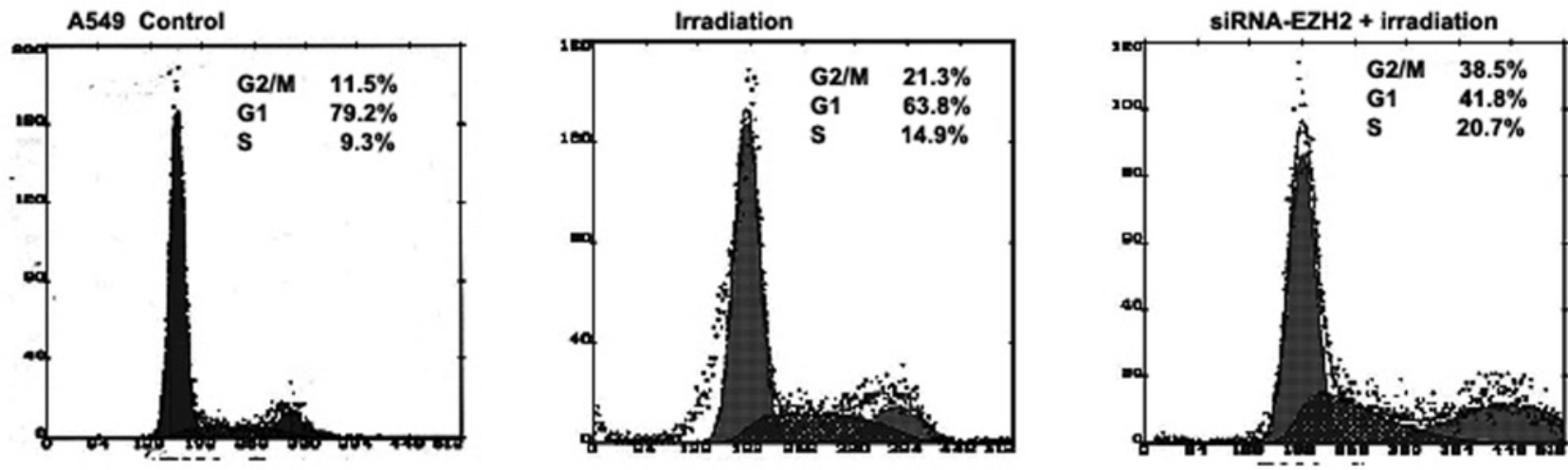

HTB56 Control
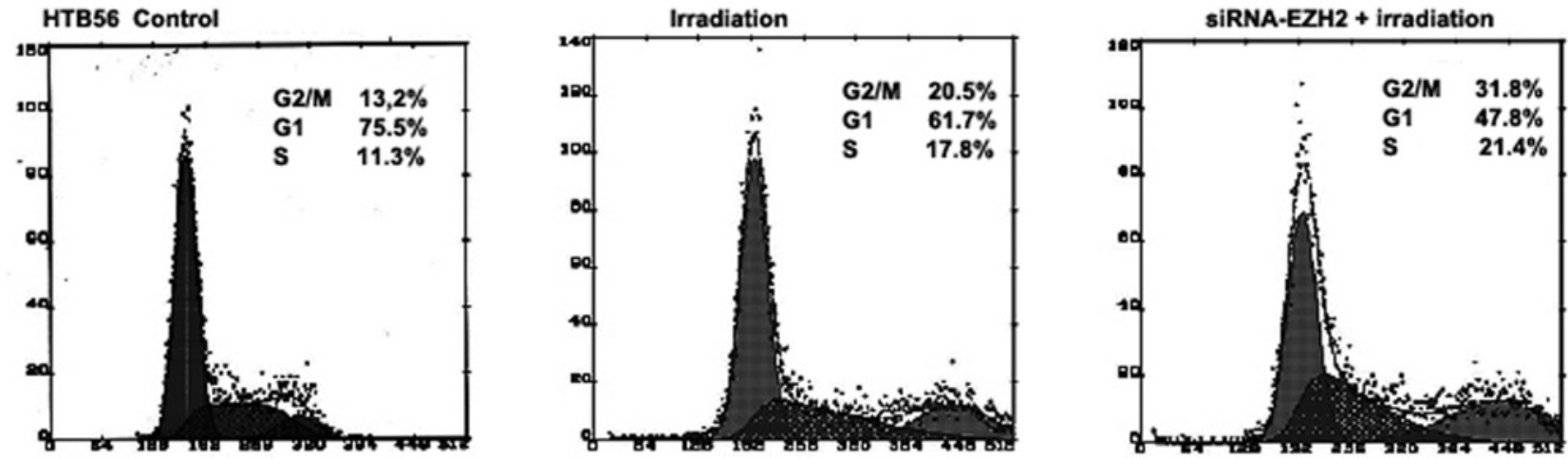

Figure 3. Effect of combination therapy on the cell cycle in A549 and HTB-56 cells. Representative profiles of an analysis of cell cycle kinetics are shown. The cells were treated with $\gamma$-ray irradiation at $\sim 2 \mathrm{~Gy} / \mathrm{min}$ in the absence and presence of siRNA-EZH2. Cell cycle analysis was performed 2 days after treatment. siRNA, small interfering RNA.
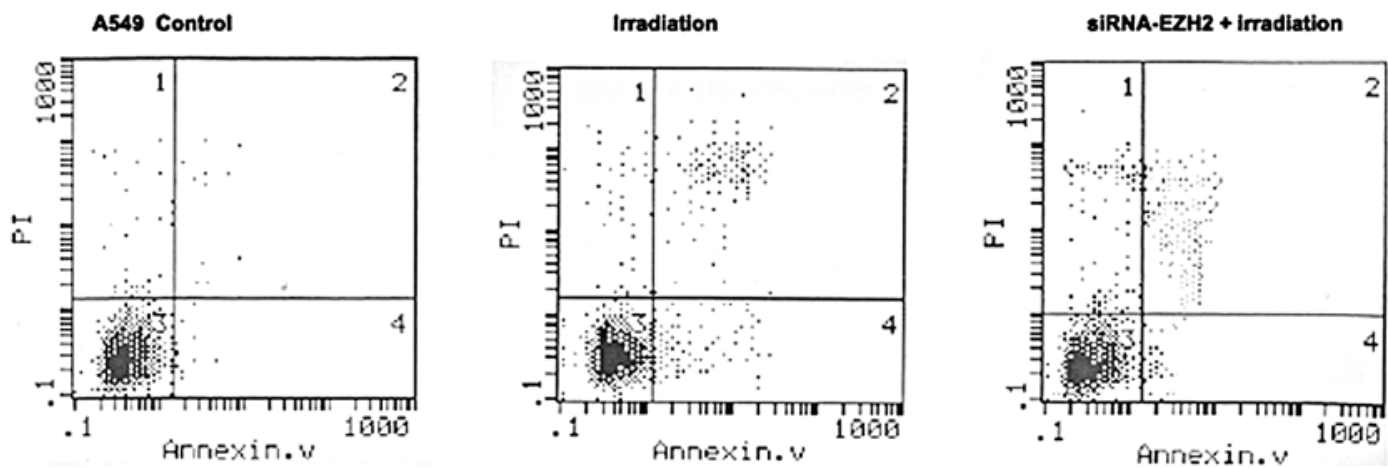

HTB56 Control

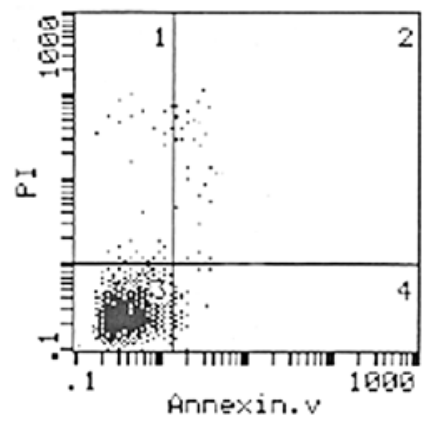

Irradiation

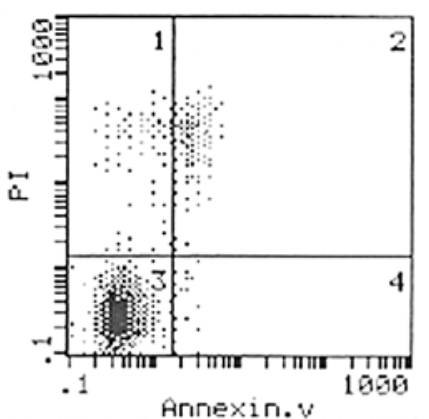

SIRNA-EZH2 + irradiation

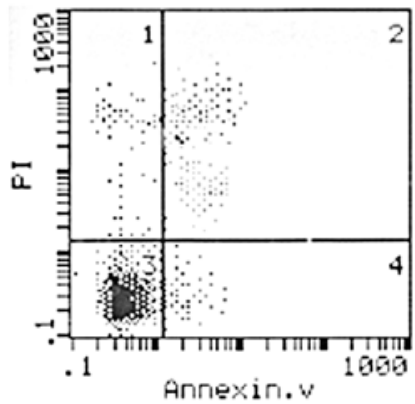

Figure 4. Effect of combination therapy on apoptosis in A549 and HTB-56 cells. The cells were evaluated by flow cytometry following treatment. The cells were treated with $\gamma$-ray irradiation at $\sim 2 \mathrm{~Gy} / \mathrm{min}$ alone and treated with irradiation in the presence of siRNA-EZH2. Cell apoptosis analysis was performed 2 days after transfection with siRNA-EZH2. siRNA, small interfering RNA; PI, propidium iodide. 

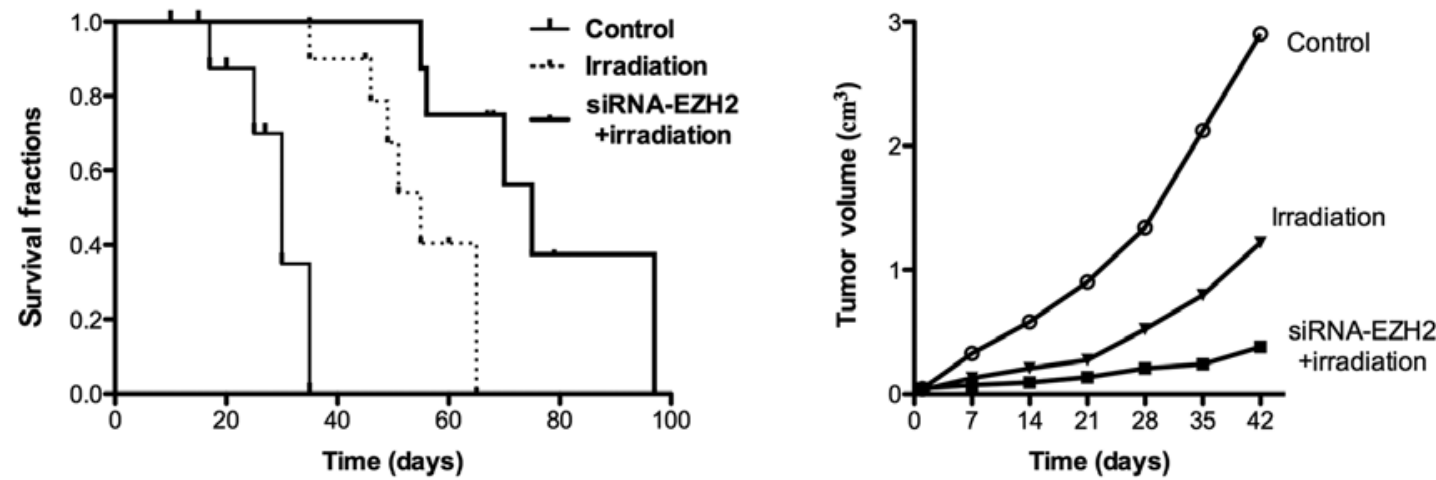

Figure 5. Therapeutic effect of irradiation combined with siRNA-EZH2 on subcutaneous human glioma tumor xenografts. Tumor volume was calculated at the indicated times as shown on the right. Tumors were measured using calipers and the statistical significance of tumor volume changes was calculated using the Student's t-test. Each time point represents the mean tumor volume for each group $(\mathrm{n}=10)$. The survival fraction following treatment is shown on the left. Irradiation in the presence of siRNA-EZH2 enhanced tumor growth inhibition and led to longer survival times than treatment with irradiation alone. siRNA, small interfering RNA.

more than 5 years remains poor. Additionally, the resistance to radiation from different lung cancer types limits the therapeutic efficacy of current treatments (10). Therefore, according to the rules for the development of more effective and less toxic treatments, there is a search for new modalities via molecular, biological, genetic and immunological methods with the aim of enhancing the survival and quality of life of NSCLC patients. This is an urgent challenge in current clinical cancer therapies. It has been demonstrated that EZH2 is overexpressed in a number of malignancies with aggressive forms and that the cell growth of breast cancer is significantly influenced following the knockdown of EZH2 with siRNA $(9,11)$. The purpose of the present study was to test the hypothesis that therapeutic efficiency increases following irradiation therapy in combination with siRNA-EZH2 downregulation in human A549 and HTB-56 cells in vitro and in vivo.

In the present study, following the transfection of siRNA-EZH2 into A549 and HTB-56 cells, the inhibition of cell proliferation caused by irradiation was enhanced compared with radiotherapy alone. These results markedly suggest that the reduced expression of EZH2 by siRNA treatment is closely associated with the therapeutic efficiency of irradiation on cancer cells. Furthermore, these findings demonstrate that the inhibitory efficiency of the combination treatment on cell proliferation was markedly higher in A549 cells than that in HTB-56 cells due to the different expression levels of EZH2. This observation indicates a possible reason for the divergence of the clinical irradiation therapy on NSCLC patients. Additionally, we evaluated the effect of siRNA-EZH2 on cellular sensitivity to irradiation in terms of changes in the cell cycle and cell apoptosis. Different mechanisms, including several related signaling pathways that delay or inhibit molecular transitions in cancer cells, have been investigated and found to influence cell cycle control. These control pathways are critical for genomic integrity and for the repair and survival of cells exposed to DNA-damaging agents $(6,12,13)$. We found that EZH2 silencing arrested the cells in $S$ and $G_{2} / M$ phases, delayed the cell cycle progression and increased cell apoptosis when combined with irradiation therapy. The possible mechanism of these phenomena is that the process of DNA repair was blocked with a corresponding effect on cell cycle arrest and apoptosis. This hypothesis is consistent with a previous study, which reported that EZH2 silencing with siRNA in breast cancer and ovarian cancer cells resulted in cell cycle arrest in the $\mathrm{G}_{0} / \mathrm{G}_{1}$ phase .

Based on these results, we found that the expression of EZH2 is different in A549 and HTB-56 cells. This may explain the divergent activity of radiation therapy on these cells and th reason for the treatment with siRNA inducing greater therapeutic efficiency in A549 cells compared to HTB-56 cells in terms of cell proliferation, cell cycle progression and apoptosis. This finding suggests that a higher sensitivity to irradiation therapy was induced in A549 cells after the knockdown of EZH2. These data also serve as reminders that a biological treatment, including specific or new biomarkers of lung cancer, should be personalized in the clinical therapy of NSCLC patients. Additionally, we established subcutaneous lung cancer xenografts with A549 cells in immunodeficient mice and evaluated the inhibition of tumor growth induced by combined ionizing radiation and siRNA-EZH2 treatment. We found that the combined therapy with intratumoral injections of siRNA-EZH2 into A549 tumor xenografts significantly increased the survival rate and inhibition of tumor growth $(\mathrm{P}<0.01)$ compared with radiotherapy alone.

Combination therapies using radiotherapy and biological agents to target cancer have been investigated for several years. Research on cancer cell growth signaling pathways provides potential benefits in addition to the possibility of enhancing and improving therapeutic outcomes. Such combination therapies generate more specific tumor responses and decreased toxicity to normal tissues. Additionally, partially effective therapeutic modalities may be combined without having to significantly reduce their doses to avoid treatment-related toxicities. In the present study, by defining the proposed role of EZH2 in the process of lung cancer treatment following irradiation, a new biological marker and therapeutic target were identified to demonstrate that the application of gene silencing methods with siRNA are potentially useful as a highly specific tool in the modulation of tumor-promoting effects. 
In conclusion, this study has shown that EZH2 silencing with siRNA, enhanced A549 and HTB-56 cell sensitivity to irradiation in vitro and in vivo and that this effect was influenced by the EZH2 expression level. The information obtained from the present study may be used to develop new treatments for the clinical therapy of NSCLC patients.

\section{References}

1. Fidias P and Novello S: Strategies for prolonged therapy in patients with advanced non-small-cell lung cancer. J Clin Oncol 28: 5116-5123, 2010.

2. Fuld AD, Dragnev KH and Rigas JR: Pemetrexed in advanced non-small-cell lung cancer. Expert Opin Pharmacother 11: 1387-1402, 2010.

3. Whitehurst AW, Bodemann BO, Cardenas J, Ferguson D, Girard L, Peyton M, Minna JD, Michnoff C, Hao W, Roth MG, et al: Synthetic lethal screen identification of chemosensitizer loci in cancer cells. Nature 446: 815-819, 2007.

4. Francis $\mathrm{H}$ and Solomon B: The current status of targeted therapy for non-small cell lung cancer. Intern Med J 40: 611-618, 2010.

5. Suvà ML, Riggi N, Janiszewska M, Radovanovic I, Provero $P$, Stehle JC, Baumer K, Le Bitoux MA, Marino D, Cironi L, et al: $\mathrm{EZH} 2$ is essential for glioblastoma cancer stem cell maintenance. Cancer Res 69: 9211-9218, 2009.

6. Lennes IT and Lynch TJ: Quality indicators in cancer care: development and implementation for improved health outcomes in non-small-cell lung cancer. Clin Lung Cancer 10: 341-346, 2009.
7. Yamaguchi J, Sasaki M, Sato Y, Itatsu K, Harada K, Zen Y, Ikeda H, Nimura Y, Nagino M and Nakanuma Y: Histone deacetylase inhibitor (SAHA) and repression of EZH2 synergistically inhibit proliferation of gallbladder carcinoma. Cancer Sci 101: 355-362, 2010.

8. Fujii S, Ito K, Ito Y and Ochiai A: Enhancer of zeste homologue 2 (EZH2) down-regulates RUNX3 by increasing histone H3 methylation. J Biol Chem 283: 17324-17332, 2008.

9. Tsang DP and Cheng AS: Epigenetic regulation of signaling pathways in cancer: role of the histone methyltransferase EZH2. J Gastroenterol Hepatol 26: 19-27, 2011.

10. Gonzalez ME, Li X, Toy K, DuPrie M, Ventura AC, Banerjee M, Ljungman M, Merajver SD and Kleer CG: Downregulation of EZH2 decreases growth of estrogen receptor-negative invasive breast carcinoma and requires BRCA1. Oncogene 28: 843-853, 2009.

11. Fiskus W, Wang Y, Sreekumar A, Buckley KM, Shi H, Jillella A, Ustun C, Rao R, Fernandez P, Chen J, et al: Combined epigenetic therapy with the histone methyltransferase EZH2 inhibitor 3-deazaneplanocin A and the histone deacetylase inhibitor panobinostat against human AML cells. Blood 114: 2733-2743, 2009.

12. Panousis D, Patsouris E, Lagoudianakis E, Pappas A, Kyriakidou V, Voulgaris Z, Xepapadakis G, Manouras A, Athanassiadou AM and Athanassiadou P: The value of TOP2A, EZH2 and paxillin expression as markers of aggressive breast cancer: relationship with other prognostic factors. Eur J Gynaecol Oncol 32: 156-159, 2011.

13. Tang X, Milyavsky M, Shats I, Erez N, Goldfinger N and Rotter V: Activated p53 suppresses the histone methyltransferase EZH2 gene. Oncogene 23: 5759-5769, 2004. 